\title{
Discursive influences on clinical teaching in Australian undergraduate nursing programs
}

\author{
Lisa G. McKenna ${ }^{a}$, Sally J. Wellard \\ a School of Nursing, Monash University, Peninsula Campus, Frankston, Vic. 3199, Australia \\ ${ }^{b}$ School of Nursing, University of Ballarat, Mt Helen, Vic. 3350, Australia
}

Accepted 18 December 2003, Nurse Education Today.

\begin{abstract}
Summary:
Clinical teaching is a vital, yet multidimensional component of Australian undergraduate nursing courses. Unlike other parts of curricula, clinical teaching relies on the both higher education and health care sectors to meet prescribed goals and for effective student learning to occur. As such it is influenced by discourses from within both education and health. Whilst there is considerable literature related to undergraduate nursing clinical teaching; it mainly deals with practical aspects such as effectiveness of clinical teaching or discussions of models employed. Only a small pool of literature exists that discusses the construction of clinical teaching including the factors that have influenced the development of practices both in the past and present. Using the work of Foucault, this paper examines dominant and competing discourses influencing clinical teaching through their constructions within the literature. These are discourses of academia, nursing, and economics. The discussion situates these discourses and discusses how some of the resultant issues surrounding clinical education remain largely unresolved.
\end{abstract}

Keywords: Discourse; Clinical teaching; Foucault; Nursing; Economics; Academia.

\section{Introduction}

Clinical education provides a mechanism for nursing students to extend classroom learning into nursing practice environments. Internationally, this component of education has undergone continual change and modification since the move of nurse education into the tertiary sector. This change was most significant due to the shift from clinical education being part of a service-oriented course to an academic, university-based one. Both the health care and higher education sectors have grappled with emerging issues and changing roles. Through an extensive literature review, it has been possible to identify a range of discourses that have been influential in directing the development of clinical education in undergraduate nursing programs since the transfer.

The work of Michel Foucault $(1970,1972,1975,1977,1980)$ guided the analysis of dominant discourses that have impacted on the development of clinical education. Discourses, in a Foucauldian sense, refer to more than the language we speak, they form the constraining grids that give rise to the ways in which we think and act.

... a discourse is a "system of statements which cohere around common meanings and values..." (Hollway, 1983, p. 231). Discourses construct relationships we have in and with the world: the ways we speak about the world to some extent structure our 'realities' (Drewery, 1998, p. 103).

Our ways of knowing and being in the world are not simply governed by one discourse; we are influenced by many competing discourses. By reviewing of the literature about clinical teaching in nursing three groups of discourses are revealed as dominant. These are discourses of academia (focus on the individual); discourses of nursing (focus on ideas about what nursing is or might be) and discourses of economics (focus on cost and efficiency). These dominant discourses in turn influence the practices of clinical teachers and their employers. The impact of these discourses on education-clinical practice relationships and organisational practices are reviewed leading to questions about how 
realistic current expectations of, and the overall goals developed for, undergraduate clinical education are.

\section{Education-practice relationships}

Until the latter stages of the twentieth century Australian nurse teachers were employed in positions of responsibility for both classroom and clinical education of nursing students within hospital-based apprenticeship programs where student nurses were employees of the hospital (Russell, 1990). In the decades following World War II there was a progressive increase in the numbers of classroom hours reflecting growth in scientific knowledge and increasing use of technology (Herdman, 1995). This process culminated in the transfer of nurse education to the tertiary sector where students no longer had roles as employees and education was located apart from hospital environments. The education of nurses appeared to move from a system that was driven by clinical demands to one that was driven by academic agendas and resulted in dramatic changes to the roles of nurse teachers. Learning rather than service became of primary importance for students (Peterson and Schaffer, 2001).

\section{Move to tertiary education}

Accompanying the move to tertiary educational environments academic roles became increasingly significant for nurse educators. Influenced by a range of academic discourses, nurses working in university schools of nursing assumed expanded roles that encompassed research, scholarship and community service in addition to significant teaching loads. Lesser emphasis was directed towards clinical nursing educational aspects of teachers' roles (Lee, 1996; Clifford, 1996) with more importance placed on the generation of nursing knowledge (Wong and Wong, 1987) as academic discourses began to influence the construction of nurse academic roles.

Clinical teaching was considered to be a time consuming function with little academic reward (Ludwick et al., 1998), and soon became ranked as a low priority for nurse academics (Brown et al., 1998). The competing demands of the academic role resulted in time constraints and seeking other ways of supervising students within clinical settings emerged as a strategy for managing time. The introduction of sessional clinical teachers was one response to this situation. Within this model of managing undergraduate clinical teaching, individuals are employed casually for the period of clinical placements therefore providing a more cost-effective option. However, a gradual reduction in available clinical weeks within curricula, low job security and long periods without work has impacted negatively on the recruitment and retention of sessional teachers (Napthine, 1996). Additionally, individuals employed as sessional clinical teachers are often inexperienced as teachers (Napthine, 1996; Duke, 1996; Myrick, 1991) and may not be adequately aware of curricula goals and philosophies.

Whilst academic discourses are dominant there is a simultaneous tension between the academic and the clinical. The increasing focus on academic ventures by nurse academics has subsequently led to questioning the roles and expectations of them in clinical settings, and their responsibilities for undergraduate clinical education (Cave, 1994; Clifford, 1996; Owen, 1993). Tanner (1998) suggests that it is difficult for clinicians working in clinical settings to keep pace with changes in health care practices, making it virtually impossible for academics to do the same. Adding to the tensions, a recent enquiry into nursing education (Heath, 2002) supports Cave's (1994) argument for nurse academics maintaining clinical currency if they are to continue to be able to assist students to apply theory to realistic and current practice situations.

\section{Nursing environments and clinical education}

There is evidence to suggest that the culture of nursing has an impact upon the way undergraduate clinical education is delivered. This situation differs between various clinical areas. Reid (1994) has argued that some nurses in clinical areas perceive students as a burden and "intra-nursing cultural tensions have become part of clinical education" (p.184) 
and impact on student learning. In other cases clinical staff have little understanding of the curriculum so may be poorly informed about students' learning needs, resulting in reluctance to allow students to apply their knowledge and skills by carrying out particular procedures (Napthine, 1996). These issues can in part be attributed to the short-term nature of individual clinical practicum experiences within higher education curricula, in contrast to hospital based education where nursing students were recognized as part of the permanent ward culture (Paterson, 1997).

The current context of practice also influences clinical education and will continue to have an impact (Beeman, 2001). In contrast to previous decades, patients in general wards areas are more acutely ill, whilst conversely, length of stay is reducing. Becker and Neuwirth (2002) succinctly argue that many patients who would once have been admitted to intensive care units are now managed in medical-surgical areas, whilst those who would have been located there are now cared for in the community. This has led to a reduction in the amount of attention clinicians provide students, and has contributed to a decrease in the availability of suitable learning experiences (Beeman, 2001). The increasing acuity of patients compounded with the reduced attention to students leads to potentially more hazardous clinical learning environments for students (Chamings and Payne, 1994). Furthermore, Tanner (2002) argues that changes impacting on clinical nursing practice, especially through increased patient throughput, impact negatively on students' abilities to action the plans for nursing care they have developed and may impact on learning outcomes. In the recently delivered Australian National Review of Nurse Education (Heath, 2002), this issue was recognized as one which could make addressing students' clinical learning needs more difficult in future.

\section{Working together}

Recent clinical education literature has documented the emergence of increasing collaboration between education and practice sectors. This move has been influenced by a realization that previous models of clinical education are unsustainable and an acknowledgement that, both education and health care sectors have significant roles to play in undergraduate clinical education (Atack et al., 2000). Dominant terminology includes clinical partnership, joint appointment and industry collaboration highlighting the contributions made by both sectors in educational processes. Towards the late 1990s, the notion of partnership increased in prominence within nursing with the recognition that the

present culture of clinical education by "default", unavailability of resources and few formal collaborative structures between education institutions and service providers cannot sustain the complex demands and pace of the evolution of professional nursing practice (Edmond, 2001, p. 258).

The examples of collaborative arrangements are growing with a variety of clinical partnership models. Davies et al. (1999) describe an Australian university that developed a clinical partnership model for undergraduate nursing students. The authors report that this resulted in stronger relationships and led to mutual benefits, including streamlining of clinical placements with increased rationalization of the number of agencies being utilized, along with professional recognition for registered nurses in clinical teaching roles.

Preceptor roles for supporting undergraduate nursing students learning have evolved simultaneously with the developments in collaboration and partnership. Beeman (2001) and Nordgren et al. (1998) argue the need to adopt preceptor roles in response to decreasing clinical and faculty resources. Beeman (2001) further argues for shifting some teaching responsibility onto clinicians with academic support as collaborative. In practice, preceptor models can be difficult to sustain with a potential for burnout resulting from increased workload for the preceptor. The situation is further complicated by staff shortages and high patient acuity and throughput. Within this context, the preceptor role is frequently 
undertaken by the same nurses who carry significant clinical loads and for whom there is little recognition of the extra role of preceptor (Edmond, 2001). This arguably will increase stress on students who are aiming to achieve learning objectives, and on preceptors who have responsibility for both patients and students (Coates and Gormley, 1997). Furthermore, this model may not suit the diverse learning needs of all students (Beattie, 1998).

Discourses of collaboration are also evident in areas where joint initiatives are undertaken, such as joint appointments. McKenna and Roberts (1999) argue that joint appointments may assist with bridging the theory-practice gap in clinical settings. They define joint appointments as 'a variety of arrangements whereby concurrent employment occurs within an educational institution and a clinical setting' (p. 14). Such appointments arguably provide support for students as well as leading to improved quality of patient care through academic endeavours. However, these have been historically noted as challenging to deliver (Beitz and Heinzer, 2000).

\section{Organisational practices}

The organisational practices involved in the delivery and support of undergraduate clinical teaching have been shaped by the contemporary dominance of economic discourses in both health care and educational settings. The centrality of economic discourses in health care delivery has become more visible internationally in recent decades as the demand for constantly increasing health care spending has been tempered with new models for constraining health care expenditure (Hunter, 1996).

As a consequence of the dominance of economic rationalist discourses and the systems of control introduced, the practice of nursing has been significantly influenced (Heggen and Wellard, 2003). There has been a restructuring of the workforce with an increase in part time and casual employees. This has contributed to a loss of social capital, trust and caring (Light, 2001) between the staff of health care services.

Economic discourses across both health care and tertiary education settings have been dominant. In addition, discourses of competition between universities, and between universities and health care agencies have been significant. Workplace and industrial discourses have been influential to the construction of clinical teaching roles.

\section{Economic discourses}

Throughout the literature relating to clinical education, economic discourses emerge as significant in shaping the development of clinical teaching models and roles. In a study of clinical learning programs in Australia, Wellard et al. (2000) concluded that funding cuts in both health and education sectors influenced the clinical teaching models being used, the scope of clinical experiences offered to students and the numbers of hours students spent in clinical areas. In a subsequent paper, these authors argued that imbalances exist between funding made available for undergraduate clinical education and ensuring that acceptable practice standards were maintained (Williams et al., 2001). Recognising that economic issues were significant in clinical education, Heath (2002) concludes that these issues led to an impact on the overall quality of nursing courses.

Currently discourses of economic rationalism are dominant where it is argued as less cost effective for academic staff to fulfill clinical teaching roles than using alternate models for the provision of clinical teaching. Economic discourses have been enlisted to support the casualisation of clinical teaching for students whilst on placement. Reid (1994) suggests that sessional staff are cheaper to employ than academic staff, and needed only for set blocks of time. Whilst initially considered cost effective compared with the cost of deploying academic staff to undertake clinical teaching, schools of nursing continue to experience economic constraints in the provision of clinical teaching (Heath, 2002). Furthermore, Reid (1994) predicts that the economic situation is unlikely to be assisted by industry. What is less clear is the educational impact of the employment of sessional clinical teachers. It has 
been argued that this model can conflict with curriculum goals as commonly these sessional clinical teachers possess the least teaching experience (Ludwick et al., 1998; Reid, 1994) or understanding of the curriculum so may be least prepared for assisting students apply theoretical knowledge into clinical practice.

The maintenance of fixed clinical teacher to student ratios has been a cost effective measure for optimizing the clinical budget within schools of nursing. Generally, an Australian clinical teacher is responsible for the supervision of eight students; however, this may vary slightly across institutions depending on the particular clinical focus of the experience or agency preference (Wellard et al., 2000). The more students a clinical teacher is responsible for, the more cost effective the model. Some universities are attempting to maximize expenditure by reducing the number of hours worked by clinical teachers. In some small clinical agencies where eight students cannot be accommodated, a clinical teacher's working hours are reduced accordingly. This assists with maintaining an acceptable overall cost per student for the length of the placement (Williams et al., 2001).

The increasing costs of employing sessional clinical teachers, along with difficulty in recruitment and retention of sessional teachers contributed to the development of the clinical teaching associate, and later preceptor, models for the delivery of clinical education. Subsequent to the introduction of the clinical teaching associate model, economic factors have continued to play a major role with rising salary costs increasing the remuneration expected by health care agencies to cover costs for staff seconded by the university to undertake clinical teaching. The introduction of collaborative preceptor models for clinical teaching has been partially attributed to the higher costs of other models (Bowles, 1995; Myrick, 1988). Preceptorship arrangements involve students working in a one-to-one relationship with a clinician from the host health care agency (Grealish and Carroll, 1998). The preceptor maintains normal workload, so does not need to be removed from the ward area and as such replacement costs are not needed. Whether this situation continues into the future is uncertain, however, collaborative approaches can assist with controlling cost outlays for clinical supervision and teaching (Williams et al., 2001).

\section{Competition}

A range of factors indicates that discourses of competition may be significant in shaping clinical teaching roles. Each year a number of schools of nursing vie intensely for limited placements for undergraduate nursing students across a range of clinical focus areas (Tanner, 1998). Certainly, in order to maintain the intended clinical focus for students, schools of nursing may be required to compete for those places, or secure places that may not be able to deliver the type of experiences required for students (Lumby, 1989). A shift towards securing "market share" of available places has become dominant amongst schools of nursing where competition is extending to securing relationships with health care facilities through partnership arrangements. This has been driven in part by significant emphasis being placed on inpatient acute clinical practice within nursing curricula (Grindel et al., 2001).

Competitive discourses are also evident between health care venues and schools of nursing. The National Review of Nurse Education in the Higher Sector (Reid, 1994) highlighted the issue of differing expectations of nurse education between the two sectors. Lumby (1989) suggests "competing goals within the clinical area as well as the specific philosophies and procedures on which patient and student care are based must be considered. .." (p. 299). Furthermore, classroom theory and clinical practice may not always correlate with a variety of approaches to individual nursing skills from nurse to nurse, ward to ward and agency to agency (McCaugherty, 1991). A state of competition emerges as each teaching site seeks to meet its requirements and assert itself as the more significant and important. 
Current clinical teaching models have led to a number of workplace and industrial issues in recent years. Significantly, difficulty in recruitment and retention of clinical teachers has been noted by a number of authors. This in part can be attributed to a perceived lack of value placed upon the work of clinical teachers (Duke, 1996; Schuster et al., 1997; Wong and Wong, 1987). In addition, Napthine (1996) identifies a number of negative aspects of performing the role of sessional clinical teaching. These include inadequate payments for the role performed and lack of employment security for clinical teachers employed on casual contracts that may further impact on the retention of clinical teachers.

Furthermore, whilst the recruitment of appropriate numbers of clinical teachers may not be a significant problem for many schools of nursing, recruiting nurses with the required combination of skills may well pose difficulties (Wellard et al., 2000). This may effectively result in students not being supervised by clinical teachers with the skills repertoire necessary for the actual clinical environment hosting the student and impact on learning outcomes for students.

\section{Conclusion}

Clinical teaching is complex and multidimensional. In Australia, there has been considerable change since nurse education moved into the higher education sector. The Foucaudian lens of discourse uncovers diverse influences on complex social and political constructions of clinical teaching. Discourses arising from within health and education have played a significant role in developing clinical teaching practices. The competing nature of health care and educational discourses in the provision of clinical education has meant that many issues have guided change. Nurse academics and clinicians still search for 'ideal' means for delivering cost-effective, educationally sound clinical education for undergraduate nursing students. This paper has sought to present enhanced discussion and understanding of influencing factors that may assist with future directions.

Power-knowledge relationships that arise in these discourses are not clearly understood and there is a need for further investigation of how these play out and the subsequent construction of the student. This may indicate a need for examination of specific practices which occur within clinical teaching workplaces.

\section{References}

Atack, L., Comacu, M., Kenny, R., LaBelle, N., Miller, D., 2000. Student and staff relationships in a clinical practice model: impact on learning. Journal of Nursing Education 39 (9), 387-392.

Beattie, H., 1998. Clinical teaching models: a review of the role of preceptor in the undergraduate nursing program. Australian Journal of Advanced Nursing 15 (4), 1419.

Becker, M.K., Neuwirth, J.M., 2002. Teaching strategy to maximize clinical experience with beginning nursing students. Journal of Nursing Education 41 (2), 89-91.

Beeman, R.Y., 2001. New partnerships between education and practice: precepting junior nursing students in the acute care setting. Journal of Nursing Education 40 (3), 132134.

Beitz, J.M., Heinzer, M.M., 2000. Faculty practice in joint appointments: implications for nursing staff development. Journal of Continuing Education in Nursing 31 (5), 232237.

Bowles, N., 1995. A critical appraisal of preceptorship. Nursing Standard 9 (45), 25-28.

Brown, N., Forrest, S., Pollock, L.C., 1998. The ideal role of the nurse teacher in the clinical area: a comparison of the perspectives of mental health, learning difficulties and general nurses. Journal of Psychiatric and Mental Health Nursing 5 (1), 11-19.

Cave, I., 1994. Nurse teachers in higher education - without clinical competence, do they have a future? Nurse Education Today 14 (5), 394-399.

Chamings, P.A., Payne, B., 1994. Partners in clinical teaching. Nursing Connections 7 (2), 57-61. 
Clifford, C., 1996. Nurse teacher's clinical work: a survey report. Journal of Advanced Nursing 23, 603-611.

Coates, V.E., Gormley, E., 1997. Learning the practice of nursing: views about preceptorship. Nurse Education Today 17 (2), 91-98.

Davies, E., Turner, C., Osborne, Y., 1999. Evaluating a clinical partnership model for undergraduate nursing students. Collegian 6 (2), 23-28.

Drewery, W., 1998. Unemployment: what kind of problem is it? Journal of Community and Applied Psychology 8, 101-118.

Duke, M., 1996. Clinical evaluation - difficulties experienced by sessional clinical teachers of nursing: a qualitative study. Journal of Advanced Nursing 23, 408-414.

Edmond, C.B., 2001. A new paradigm for practice education. Nurse Education Today 21, 251-259.

Foucault, M., 1970. The Order of Things: An Archaeology of the Human Sciences. Random House, New York.

Foucault, M., 1972. The Archaeology of Knowledge. Tavistock, New York.

Foucault, M., 1975. The Birth of the Clinic: An Archaeology of Medical Perception. Vintage, New York.

Foucault, M. 1977. Discipline and Punish: the Birth of the Prison. Foucault, M., 1980. Power/Knowledge: Selected Interviews and Other Writings 1972-1977. Harvester Press, London.

Grealish, L., Carroll, G., 1998. Beyond preceptorship and supervision: a third clinical teaching model emerges for Australian nursing education. Australian Journal of Advanced Nursing 14 (2), 24-30.

Grindel, C.G., Bateman, A.L., Patsdaughter, C.A., Babington, L.M., Medici, G., 2001. Student contributions to clinical agencies: a comparison of adult health and psychiatric staff nurses' perceptions. Nursing and Health Care Perspectives 22 (4), 197-202.

Heath, P., 2002. National Review of Nursing Education 2002 - Our Duty of Care. Commonwealth of Australia, Canberra.

Heggen, K., Wellard, S.J. 2003. Increased unintended patient harm in nursing practise as a consequence of the dominance of medical discourses, In press, International Journal of Nursing Studies.

Herdman, E., 1995. Professionalisation, rationalisation and policy change: the transfer of pre-registration nurse education to the university sector. In: Short, S.D. (Ed.), Annual Review of Health Social Sciences, Health Policy, vol. 5. University of New South Wales, Sydney.

Hunter, D.J., 1996. The changing roles of health care personnel in health and health care management. Social Science \& Medicine 43 (5), 799-808.

Lee, D.T.F., 1996. The clinical role of the nurse teacher: a review of the dispute. Journal of Advanced Nursing 23, 1127-1134.

Light, D.W., 2001. Comparative institutional response to economic policy managed competition and governmentality. Social Science and Medicine 52, 1151-1166.

Ludwick, R., Dieckman, B.C., Herdtner, S., Dugan, M., Roche, M., 1998. Documenting the scholarship of clinical teaching through peer review. Nurse Educator 23 (6), 17-20.

Lumby, J., 1989. Preparation for practice. In: Gray, G., Pratt, R. (Eds.), Issues in Australian Nursing, vol. 2. Churchill Livingstone, Melbourne.

McCaugherty, D., 1991. The theory-practice gap in nurse education: its causes and possible solutions. Findings from an action research study. Journal of Advanced Nursing 16, 1055-1061.

McKenna, B., Roberts, R., 1999. Bridging the theory-practice gap. Kai Tiaki Nursing New Zealand 5 (2), 14-16.

Myrick, F., 1988. Preceptorship: a viable alternative clinical teaching strategy? Journal of Advanced Nursing 13, 588-591.

Myrick, F., 1991. The plight of clinical teaching in baccalaureate nursing education. Journal of Nursing Education 30 (1), 44-46. 
Napthine, R., 1996. Clinical education: a system under a pressure. Australian Nursing Journal 3 (9), 20-24.

Nordgren, J., Richardson, S.J., Laurella, V.B., 1998. A collaborative preceptor model for clinical teaching of beginning nursing students. Nurse Educator 23 (3), 27-32.

Owen, S., 1993. Identifying a role for the nurse teacher in the clinical area. Journal of Advanced Nursing 18 (5), 816-825.

Paterson, B.L., 1997. The negotiated order of clinical teacher. Journal of Nursing Education 36 (5), 197-205.

Peterson, S.J., Schaffer, M.A., 2001. Service-learning: isn't that what nursing education has always been? Journal of Nursing Education 40 (2), 51-52.

Reid, J. 1994. Nursing Education in Australian Universities: Report of the National Review of Nurse Education in the Higher Education Sector - 1994 and beyond.

Commonwealth Department of Human Services and Health, Australian Government Publishing Service, Canberra.

Russell, R.L., 1990. From Nightingale to Now: Nurse Education in Australia. Harcourt Brace Jovanovich, Sydney.

Schuster, P., Fitzgerald, D.C., McCarthy, P.A., McDougal, D., 1997. Work load issues in clinical nursing education. Journal of Professional Nursing 13 (3), 154-159.

Tanner, C.A., 1998. Tending to our clinical education. Journal of Nursing Education 37 (8), 335-336.

Tanner, C.A., 2002. Clinical education, circa 2010. Journal of Nursing Education 41 (2), 5152.

Wellard, S.J., Williams, A., Bethune, E., 2000. Staffing of undergraduate clinical learning programs in Australia. Nurse Education Today 20, 548-554.

Williams, A.F., Wellard, S.J., Bethune, E., 2001. Assessing Australian undergraduate clinical learning. Collegian 8 (4), 9-13.

Wong, J., Wong, S., 1987. Towards effective clinical teaching in nursing. Journal of Advanced Nursing 12, 505-513. 